\title{
Tumor neuroendocrino primario mediastinal
}

\section{Mediastinal primary neuroendocrine tumor}

\author{
Hugo Michael García-Ramos,' \\ Carlos Luque-Quispe, ${ }^{2}$ \\ Sonia Indacochea-Cáceda ${ }^{3}$
}

\author{
García-Ramos H, Luque-Quispe C, Indacochea-Cáceda S. Tumor \\ neuroendocrino primario mediastinal: Rev Soc Peru Med \\ Interna. 2020;33(4): 168-170. \\ https://doi.org/10.36393/spmi.v33i4.566
}

\begin{abstract}
RESUMEN
Se presenta el caso de un paciente varón de 49 años sin antecedentes relevantes ni hábitos nocivos, con dolor toráxico, disnea a leves esfuerzos y una masa mediastinal anterior. Fue operado, con patología e inmunohistoquímica compatible con carcinoma neuroendocrino de células grandes primario mediastinal. El tratamiento fue completado con quimioterapia más radioterapia, con buena evolución clínica.
\end{abstract}

Palabras claves: tumor mediastinal, mediastino anterior, tumor neuroendocrino.

\begin{abstract}
This the case of a 49-year-old male patient with no relevant history or harmful habits, chest pain, light-effort dyspnoea and anterior mediastinal mass. He was operated on, with pathology and immunohistochemistry compatible with primary large cells neuroendocrine carcinoma of mediastinum. Treatment was completed with chemotherapy and radiation therapy, with good clinical evolution.
\end{abstract}

Keywords: mediastinal tumor, anterior mediastinum, neuroendocrine tumor.

\section{INTRODUCCIÓN}

Los tumores neuroendocrinos (TNE) son una familia heterogénea de neoplasias que derivan de las células enterocromafinEs que forman parte del sistema endocrino difuso (APUD). ${ }^{1}$ Los TNE primarios mediastinales fueron descritos por primera vez, en 1904. ${ }^{2}$ Representan el 2-4\% de los tumores del mediastino

I Médico residente de medicina interna. Universidad Peruana Cayetano Heredia. Hospital Nacional EsSalud Edgardo Rebagliati Martins. Lima, Perú.

2 Médico residente de neumología. Universidad Peruana Cayetano Heredia. Hospital Nacional EsSalud Edgardo Rebagliati Martins. Lima, Perú.

3 Médico internista. Servicio de medicina interna, Hospital Nacional EsSalud Edgardo Rebagliati Martins. Lima, Perú. Facultad de Medicina, Universidad Ricardo Palma, Lima, Perú. anterior con una incidencia de 1 por cada 5 millones de personas. Según la clasificación de la Organización mundial de la salud (OMS), en $2015^{3}$, estos se dividen morfológicamente en carcinoide típico (CT) o de bajo grado y carcinoide atípico (CA) o de grado intermedio, ambos con una frecuencia del $8 \%$; y carcinomas neuroendocrinos (CNE) o de alto grado que a su vez se subdividen en células grandes (CNECG) y de células pequeñas (CNECP) que representan el $12 \%$ y $80 \%$ respectivamente.

Reportamos el caso de un paciente con tumor neuroendocrino de células grandes primario mediastinal.

\section{PRESENTACIÓN DEL CASO}

Paciente varón de 49 años, natural de Lima, sin antecedentes patológicos relevantes ni familiares, ni hábito de tabaco. Con tiempo de enfermedad de un año caracterizado por dolor torácico a nivel de $2^{\circ}$ y $3^{\circ}$ arco costal derecho que se irradiaba a región subescapular ipsilateral, asociado a adormecimiento a nivel de hombro y brazo derecho. Un mes antes del ingreso, refirió que se agregó disnea a moderados esfuerzos, motivo por el cual ingresó por el servicio de emergencia.

Al ingreso, los signos vitales se encontraban estables y en el examen físico mostró un leve eritema facial y edema en esclavina sin circulación colateral, y sin evidencia de alteraciones a la auscultación pulmonar y cardiaca. No presentó otros hallazgos clínicos relevantes

El hemograma y la bioquímica sanguínea no mostraron alteraciones. Los marcadores tumorales se encontraron en rangos normales (AFP: $3,4 \mathrm{ng} / \mathrm{ml}(0-10,9)$ CEA: $1,86 \mathrm{ng} / \mathrm{ml}(0-3)$, beta 2 microglobulina: 1,4mg/1 (0,6-2.1), LDH: 160u/1 (120-246), beta HCG: $<2 \mathrm{mlu} / \mathrm{ml}(<10)$, CYFRA 21.1: 2ng/ml (0-3). 
En la radiografía y tomografía de tórax, se evidencio una lesión heterogénea de bordes irregulares en el mediastino anterior, de 86 x $50 \mathrm{~mm}$, con captación del contraste y con leve realce periférico,

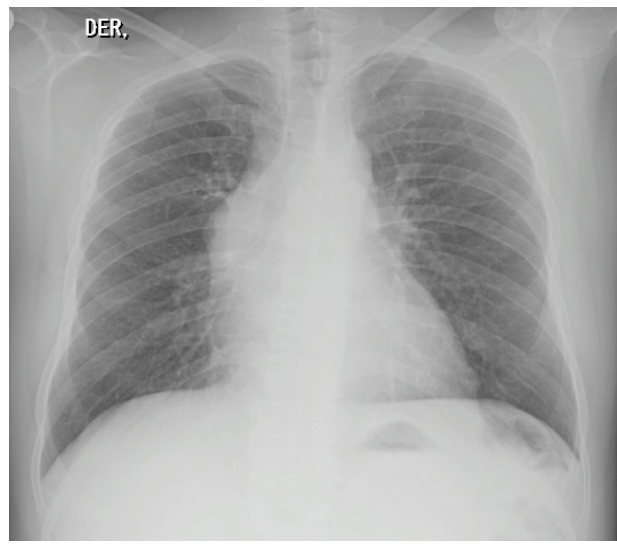

Figura I. Rx. de tórax: masa en el mediastino anterior. sin compromiso vascular ni adenopatías mediastinales (Figuras $1,2 \mathrm{~A}$ y $2 \mathrm{~B})$.

Con estos resultados se decidió programar una mediastinoscopia más resección del tumor. Los hallazgos macroscópicos fueron de una tumoración de $10 \times 6 \times 3 \mathrm{~cm}$, encapsulada, irregular, pardo oscuro con áreas amarillentas y de consistencia fibroelástica. La microscopía describió una neoplasia de aspecto epitelial de patrón organoide con áreas extensas de necrosis, con índice mitótico de $17 / 2 \mathrm{~mm}^{2}$; inmunihistoquímica de CD 56, sinaptofisina y cromogranina positivas, TTF1 y CK7 negativos, con un ki-67 del $20 \%$, compatible con carcinoma neuroendocrino de células grandes de alta grado primario mediastinal (Figuras 3A y 3B). Recibió tratamiento adyuvante con seis ciclos de cisplatino más etopósido, y 20 sesiones de radioterapia. Seis meses después, se le realizó controles de imágenes y PET-SCAN con 18-fluordesoxiglucosa, sin signos de recurrencia local, siendo dado de alta para seguimiento por consultorio externo (Figuras $4 \mathrm{~A}$ y $4 \mathrm{~B})$.
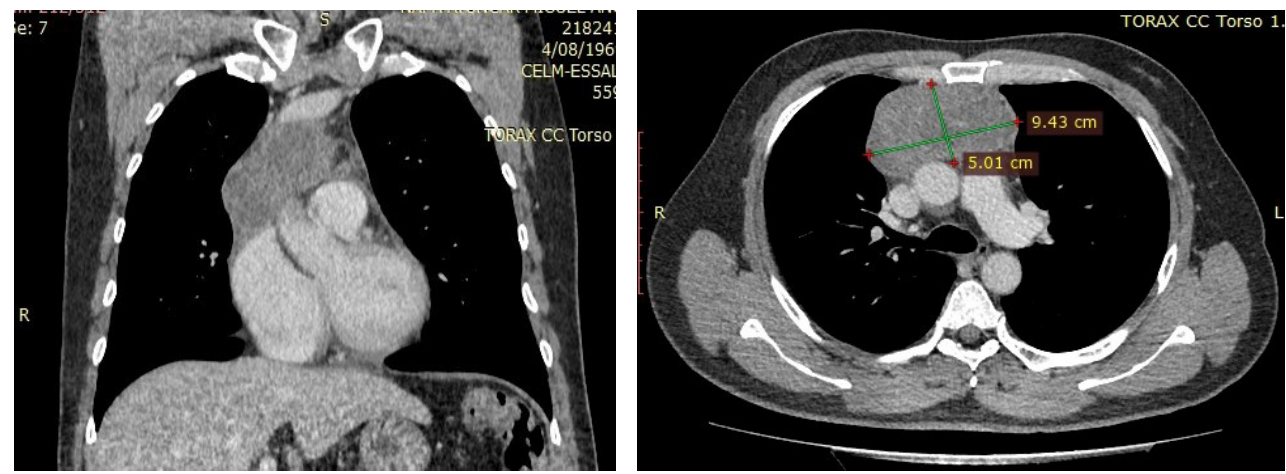

Figura 2. Tomografía preoperatoria ( $2 \mathrm{~A}$, transversal y $2 \mathrm{~B}$, coronal): masa del mediastino anterior.
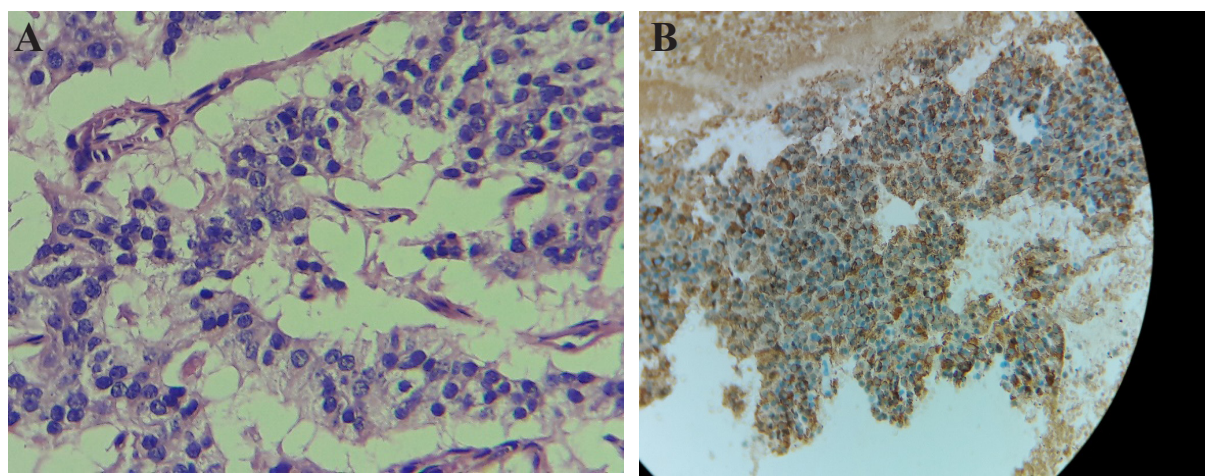

Figuras 3. 3A. Tumor neuroendocrino, células epiteliales grandes formando organoides. 3B. Sinaptofisina CD56 positivo.
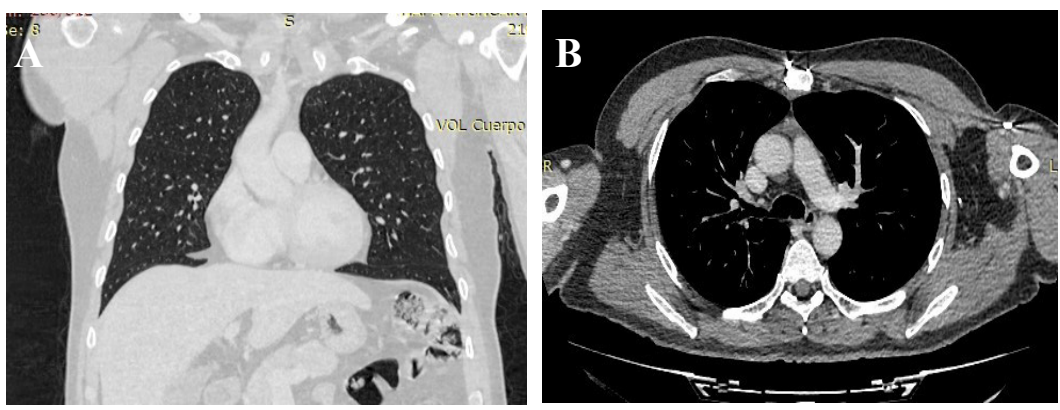

Figura 4. Tomografía postoperatoria (4A, transversal y 4B, coronal). 


\section{DISCUSIÓN}

Los tumores neuroendocrinos primarios mediastinales son un grupo raro de neoplasias, siendo los carcinomas neuroendocrinos (CNE) el grupo más frecuente. La edad media de presentación es de 51 años, así mismo los hombres son el sexo más afectado con una relación de 3 a 1 y el factor de riesgo más asociado es el hábito de tabaco. ${ }^{4}$ Dentro de la presentación clínica, pueden ser asintomáticos o presentar síntomas relacionados con el crecimiento local como dolor torácico, disnea y síndrome de vena cava superior (50\%), como sucedió con nuestro paciente..$^{5-6}$

Para el diagnóstico de este tipo de tumores se puede emplear exámenes de imágenes como la radiografía tórax, siendo los signos radiológicos más importantes el ensanchamiento del mediastino superior y la obliteración del espacio retroesternal en la vista lateral, que presento nuestro caso. En la tomografía toráxica, los hallazgos más frecuentes son la presencia de masas mediastinales anteriores de bordes ovalados sin broncograma aéreo con o sin afectación pulmonar, con áreas de necrosis y realce heterogéneo que lo diferencian de otras lesiones, siendo la visualización del hilio pulmonar a través del tumor (signo de hilio superpuesto) un parámetro para valorar el compromiso vascular. Así mismo, puede haber calcificaciones en el 30\% y asociarse a adenopatías hiliares y mediastinales en un 27\%. El PET-SCAN con 18-fluor desoxiglucosa es otra herramienta útil debido a su alta actividad metabólica para los $\mathrm{CNE} \cdot{ }^{7-8}$ Finalmente, la toma de biopsia, puede ser mediante broncoscopia, vía percutánea y abordaje quirúrgico.

Con respecto a la patología, la presencia de marcadores como la cromogranina A, el CD 56 y la sinaptofisina permiten diferenciarlos de otras estirpes tumorales. Según su índice de proliferación (ki-67) se pueden subdividir en bien diferenciados, los cuales incluyen a los G1 (bajo grado) o G2 (grado intermedio), y los pobremente diferenciados o G3 (alto grado). ${ }^{9}$ Los carcinomas se caracterizan por una tasa mitótica alta ( $>10$ mitosis $/ 2 \mathrm{~mm}^{2}$, y áreas de necrosis extensa) y ki-67 mayor al $20 \%{ }^{7,8}$ En este caso reportamos un carcinoma neuroendocrino de células grandes primario mediastinal que se caracteriza por la presencia de células con citoplasma eosinofílico y nucléolos prominentes, de un tamaño celular superior a tres veces el diámetro de un linfocito (lo cual lo diferencia del CNECP), con distribución en rosetas o cestos y con un patrón organoide de crecimiento trabecular. ${ }^{10-11}$ Dentro de los principales diagnósticos diferenciales tenemos al timoma, el cual es el tumor de mediastino anterior más frecuente y el carcinoma neuroendocrino primario pulmonar, siendo la inmunohistoquímica útil para diferenciarlos, PAX8 (+) y TTF1 (-) para el primario mediastinal. Otras neoplasias a considerar serían los otros tumores neuroendocrinos mediastinales como el carcinoide típico y atípico, los cuales son de crecimiento lento y bajo índice mitótico $\left(<10\right.$ mitosis $\left./ 2 \mathrm{~mm}^{2}\right)$. Dentro de los sitios más frecuentes de metástasis tenemos al pulmón, hígado, ósea y cerebro en $35 \%$. $^{12-14}$

En cuanto al tratamiento, si no hay compromiso del parénquima pulmonar, la mediastinoscopia más resección tumoral es el abordaje inicial ideal, con una tasa de recaída entre 5\% al 30\%, siendo recomendado en estos casos, dar terapia adyuvante de quimioterapia con cisplatino más etopósido por 4 a 6 ciclos asociado a radioterapia. ${ }^{12-14} \mathrm{Con}$ respecto al pronóstico, los de bajo grado generalmente son diagnosticados en estadio tempranos con un supervivencia superior al $90 \%$; por otro lado, los tumores de alto grado suelen ser diagnosticados en estadios avanzados y son tributarios de manejo sistémico. Los CNECG presentan una tasa de supervivencia a 5 años del 30 a $60 \% .^{15}$
En conclusión, dentro de los tumores del mediastino anterior, los neuroendocrinos primarios mediastinales son una causa poco frecuente, generando escasos síntomas por compresión local y afectación vascular, y el tumor de células gigantes es el segundo subtipo en frecuencia con un pronóstico que depende de la detección precoz, como en nuestro caso en la cual la cirugía más adyuvancia fue el tratamiento ideal.

\section{REFERENCIAS BIBLIOGRÁFICAS}

1. Annette Fisseler-Eckhoff and Melanie Demes. Neuroendocrine tumors of the lung. Cancers 2012, 4, 777-798; doi:10.3390/ cancers 4030777

2. Boubacar E, Atsame-Ebang G, Rabiou S, et al. Thymic large cell neuroendocrine carcinoma - a rare and aggressive tumor: a case report. J Med Case Rep. 2017;11(1):155. Published 2017 Jun 12. doi:10.1186/s13256-017-1331-2

3. Travis WD, Brambilla E, Nicholson AG, y col. Clasificación de 2015 de la Organización Mundial de la Salud de los tumores pulmonares: impacto de los avances genéticos, clínicos y radiológicos desde la clasificación de 2004. J Thorac Oncol. 2015; 10: 1243-60.

4. H Bohnenberger et al. Neuroendocrine tumors of the thymus and mediastinum. J Thorac Dis 2017;9(Suppl 15): S1448-S1457.

5. Travis WD. Advances in neuroendocrine lung tumors. Ann Oncol. 2010; Suppl.7:vii65-71

6. Kosmidis PA. The spectrum of carcinoids tumors. In: Pass HI, Carbone DP, John-son DH, Minna DH, Turrisi AT, editors. Lung cancer. Principles and practice. Philadelphia: Lippincott, Williams and Wilkins; 2010. p. 971-9.

7. Berman K, et al. Primary neuroendocrine tumor of the thymus: radiological and pathological correlation. Radiology case. 2020 Jan; 14 (1): 1-11

8. Kayani I, Conry BG, Groves AM, Win T, Dickson J, Caplin M, et al. A comparisonof68Ga-DOTATATE and18F-FDG PET/CT in pulmonary neuroendocrine tumors. J Nucl Med. 2009;50: 1927-32.

9. Hiroshima K, Mino-Kenudson M. Update on large cell neuroendocrine carcinoma. Transl Lung Cancer Res. 2017;6(5):530 539. doi:10.21037/tlcr.2017.06.12

10. Iyoda A, et al. Neuroendocrine tumors of the lung: clinicopathological and molecular features. Surg Today. 2020 Mar 19. doi: 10.1007/ s00595-020-01988-7.

12. Ahn S, Lee JJ, Ha SY, Sung CO, Kim J, Han J. Clinicopathological analysis of 21 thymic neuroendocrine tumors. Korean J Pathol. 2012;46(3):221-5. doi: 10.4132/ KoreanJPathol.2012.46.3.221. PubMed PMID: 23110006, PubMed Central PMCID: PMC3479770, Epub 2012 Jun 22.

13. Gaur P, Leary C, Yao JC. Thymic neuroendocrine tumors: a SEER database analysis of 160 patients. Ann Surg. 2010;251(6):1117-21. doi: 10.1097/ SLA.0b013e3181dd4ec4. PubMed PMID: 20485130.

14. Song Z, Zhang Y. Primary neuroendocrine tumors of the thymus: clinical review of 22 cases. Oncol Lett. 2014;8(5):2125-9. PubMed PMID: 25295099, PubMed Central PMCID: PMC4186590, Epub 2014 Sep 1.

15. Waghray A. Mediastinal mass in a 25-year-old man. Chest. 2014 Aug;146(2):e47-e51. doi: 0.1378/chest.13-1444.

16. Takeaki Hidaka et al. Large Cell Neuroendocrine Carcinoma of the Mediastinum Successfully Treated with Systemic Chemotherapy after Palliative Radiotherapy. Intern Med Advance Publication. The Japanese Society of Internal Medicine. doi: 10.2169/ internalmedicine.0437-17

\section{CORRESPONDENCIA:}

Hugo Michael García-Ramos

migara_12@hotmail.com

Fecha de recepción: 09.09-2020.

Fecha de aceptación: I6-II-2020. 